\title{
Nanoparticle-based Photodynamic Therapy on non-melanoma skin cancer
}

\author{
F. Fanjul-Vélez*, J. L. Arce-Diego* \\ Applied Optical Techniques Group, TEISA Department, University of Cantabria \\ Av de los Castros s/n, 39005 Santander (Spain)
}

\begin{abstract}
There are several advantages of Photodynamic Therapy (PDT) for nonmelanoma skin cancer treatment compared to conventional treatment techniques such as surgery, radiotherapy or chemotherapy. Among these advantages its noninvasive nature, the use of non ionizing radiation and its high selectivity can be mentioned. Despite all these advantages, the therapeutic efficiency of the current clinical protocol is not complete in all the patients and depends on the type of pathology. An adequate dosimetry is needed in order to personalize the protocol. There are strategies that try to overcome the current PDT shortcomings, such as the improvement of the photosensitizer accumulation in the target tissue, optical radiation distribution optimization or photochemical reactions maximization. These strategies can be further complemented by the use of nanostructures with conventional PDT.

Customized dosimetry for nanoparticle-based PDT requires models in order to adjust parameters of different nature to get an optimal tumor removal. In this work, a predictive model of nanoparticle-based PDT is proposed and analyzed. Dosimetry in nanoparticle-based PDT is going to be influenced by photosensitizer-nanoparticle distribution in the malignant tissue, its influence in the optical radiation distribution and the subsequent photochemical reactions. Nanoparticles are considered as photosensitizer carriers on several types of non-melanoma skin cancer. Shielding effects are taken into account. The results allow to compare the estimated treatment outcome with and without nanoparticles.
\end{abstract}

Keywords: Photodynamic Therapy, non-melanoma skin cancer, nanoparticles, photochemical interaction, photodynamic treatment

\section{INTRODUCTION}

Photodynamic Therapy (PDT) is an optical treatment technique employed in several clinical areas. Its aim is to destroy malignant tissues. It consists of the administration of a photosensitive substance, which is activated by the subsequent irradiation of the tumoral area. As a consequence reactive oxygen species are produced and destroy the cancerous cells [1]. However, despite its many advantages, the development of dosimetry for PDT remains a challenge due to the high variability of the treatment outcome on the basis of complex photophysical and photochemical processes [2]. Besides the limited penetration of optical radiation into the biological tissues, there are several shortcomings associated with the photosensitizers such as the hydrophobic nature of most of them or their poor selectivity among others. Emerging strategies to overcome these problems and provide an effective delivery of the photosensitizer to the target tissue include the use of nanoparticles (NPs) as an alternative to conventional molecular dye type photosensitizers [3]. In this approach, the NPs carry photosensitizer molecules either encapsulated or covalently attached to their surface [4].

Gold NPs have attracted considerable interest because of their ease of synthesis, biocompatibility and their optical properties dependence with size and shape [5,6], or even temperature [7]. There are several reports that show that gold NP conjugates are an excellent vehicle for the delivery of surface bound hydrophobic photosensitizers [8,9]. However, most of them are focused on the synthesis of gold NPs conjugates to improve the drug delivery in vitro. Therefore, relevant dosimetric factors with direct consequences on treatment outcome, such as the NPs distribution or the light propagation at tissue level, are ignored. In this context, the administration of gold NPs as carriers for conventional photosensitizers could affect the absorption of optical radiation in the biological media and therefore affect the currently limited penetration of light in the target tissue. So the effective penetration depth of light and photodynamic treatment could be even more limited in spite of photosensitizer delivery improvements.

*fanjulf@unican.es; arcedj@unican.es; phone+34 942206730; www.teisa.unican.es/toa

Optical Methods for Tumor Treatment and Detection: Mechanisms and Techniques in Photodynamic Therapy XXVII, edited by David H. Kessel, Tayyaba Hasan, Proc. of SPIE Vol.

10476, $1047609 \cdot$ @ 2018 SPIE · CCC code: 1605-7422/18/\$18 · doi: 10.1117/12.2290145 
In this work the administration of nanoparticle in PDT is studied. The study is based on light irradiation and the influence in the dosimetry when nanoparticles are present. This analysis requires a complex model to interpret the photochemical procedure involved. Nanoparticles alter light distribution in tissues, as long as the photodynamic dose, as they supply amounts of photosensitizer. This influence depends on parameters such as nanoparticles concentration, shape or material, as long as on the photosensitizer type, irradiation wavelength and irradiance. Due to the limits of optical techniques regarding penetration depth, this parameter is fundamental in the estimation of the treatment outcome. The next section shows the complex model employed for the analysis. Section 3 includes results and discussion, and section 4 contains the conclusions of the work.

\section{PHOTODYNAMIC PROCESS}

The first issue to be solved in trying to understand the photodynamic process is light propagation. Light propagation in a biological tissue implies dealing with an heterogeneous medium. This analysis does not allow an analytic exact approach of the radiation pattern with Maxwell equations. In this type of medium, the scattering is dominant over absorption and therefore both the Radiation Transport Theory (RTT) and the Diffusion Equation can be employed for this purpose [1]. The Radiation Transport Theory (RTT) allows to obtain the light distribution assuming that the scattering events are sufficiently numerous as to the light to be considered incoherent, in such a way that polarization or interference effects can be neglected. The basic idea in order to write the differential radiation transport equation is that radiation from a particle attenuates due to absorption and scattering and also gains power because another particle can scatter light in the direction of the particle of interest. This, with no sources inside the tissue and in a steady-state situation, can be written as in (1). The basic parameter of light is the specific intensity $I(\vec{r}, \hat{s})$ defined as the light power per unit area per unit solid angle. The radiation is expected to be at the point $\vec{r}$, and to follow the direction $\hat{s}$. The scattering events are treated according to the scattering phase function $p\left(\hat{s} \cdot \hat{s}^{\prime}\right)$, which contains the probabilities of light to be scattered in the different directions. Optical radiation comes from direction $\hat{s}^{\prime}$ and is redirected to $\hat{s} . \mu_{a}$ and $\mu_{s}$ stand for the absorption and scattering coefficients respectively.

$$
\hat{s} \cdot \bar{\nabla} I(r, \hat{s})=-\left(\mu_{a}+\mu_{s}\right) I(r, \hat{s})+\frac{\mu_{s}}{4 \pi} \int_{4 \pi} p\left(\hat{s}^{\prime} \cdot \hat{s}^{\prime}\right) I\left(r, \hat{s}^{\prime}\right) d \Omega^{\prime}
$$

The Monte Carlo method has demonstrated its applicability and accuracy for the numerical analysis of the radiation transport equation compared with the exact solutions. The implementation of the Monte Carlo model is multi-layered, so it is possible to define several layers of different materials with their borders perpendicular to the laser beam, which is very useful due to tissues can be usually divided in different strata. The optical characteristics and dimensions of each tissue layer are required to get a proper definition of the model. The optical parameters needed are the index of refraction $n$, the absorption and scattering coefficients and the anisotropy of scattering $g$.

When the biological media has NPs embedded, their optical properties should be taken into account to obtain the light distribution. Thus optical absorption and scattering properties of gold NPs can be obtained as expressed in (2) from the respective efficiency factor, equations 3 to 5 , calculated by means of Mie's theory for an homogeneous spherical NP taking into account its radius $r$ and the complex refractive index of gold $[10,11]$. The subscript $i$ stands for absorption or scattering, $N$ is the number of NPs per unit volume, $a_{n}$ and $b_{n}$ are amplitude coefficients for the scattered field and involve spherical Bessel functions and $x=k r$ is the size parameter, $k=2 \pi / \lambda$ is the wave number and $\lambda$ is the wavelength. These efficiency factors have a strong dependence with the optical radiation wavelength as well as with the NP size.

$$
\begin{gathered}
\mu_{i}=\pi r^{2} Q_{i} N \\
Q_{\text {ext }}=\frac{2}{x^{2}} \sum_{n=1}^{\infty}(2 n+1) \operatorname{Re}\left(a_{n}+b_{n}\right) \\
Q_{s c a}=\frac{2}{x^{2}} \sum_{n=1}^{\infty}(2 n+1)\left(\left|a_{n}\right|^{2}+\left|b_{n}\right|^{2}\right) \\
Q_{a b s}=Q_{e x t}+Q_{a b s}
\end{gathered}
$$


Once the photosensitizer accumulation and light distribution are known, the interaction of both elements with the ground state oxygen via a reaction known as Type II triggers a photochemical reaction responsible for singlet oxygen production. This kind of reactive oxygen is considered as the cytotoxic element in charge of killing the carcinogenic cells. Finally the photochemical reactions that take place during PDT can be modeled by means of a differential equations system [12,13], equations 6 to 11. This photochemical model is based in the Jablonski diagram for the generation of singlet oxygen in a type II reaction and takes into account the electronic transitions of the molecular components involved in the photodynamic procedure such as the photosensitizer or the oxygen. Its solutions permit to analyze the temporal evolution of the main molecular components concentrations such as the photosensitizer in ground state, $S_{0}$, in singlet excited state, $S_{1}$ and in triplet excited state $T$, the oxygen in ground state, ${ }^{3} O_{2}$, singlet oxygen ${ }^{1} O_{2}$ and the intracellular singlet oxygen receptors, $R$. In these equations, $\tau 1$ is the relaxation time from state $S_{1}$ to $S_{0}, \tau 3$ is the relaxation time from state $T$ to $S_{0}, \tau 0$ is the relaxation time from state ${ }^{1} O_{2}$ to ${ }^{3} O_{2}, \eta_{10}$ is the quantum yield of the transition from state $S_{1}$ to $S_{0}, \eta_{13}$ is the quantum yield of the transition from $S_{1}$ to $T, \eta_{30}$ is the quantum yield of $T$ transition to $S_{0}, \eta_{0}$ is the quantum yield of ${ }^{1} O_{2}$ transition to ${ }^{3} O_{2}, \alpha s$ is the efficiency factor for energy transfer from $T$ to ${ }^{3} \mathrm{O}_{2}, k p b$ stands for the biomolecular photobleaching rate, $k c x$ is the biomolecular cytotoxicity rate, $k s c$ is the rate of reaction of ${ }^{1} O_{2}$ with various oxygen scavengers, $v$ is light speed in tissue, $\rho$ is the photon density present at a point, $\sigma_{p s a}$ is the absorption cross-section of $S_{0}$ molecules, $P$ is the rate of oxygen diffusion and perfusion and $U$ is the cell damage repair rate. The stiff differential equations system was solved by means of a differential equation solver (ode15s) within the Matlab ${ }^{\circledR}$ platform. It was necessary to adjust relative and absolute error tolerances in order to obtain coherent results.

$$
\begin{gathered}
\frac{d\left[S_{0}\right]}{d t}=-v \rho \sigma_{p s a}\left[S_{0}\right]-k p b\left[{ }^{1} O_{2}\right]\left[S_{0}\right]+\frac{\eta_{10}}{\tau 1}\left[S_{1}\right]+\frac{\eta_{30}}{\tau 3}[T]+\frac{\alpha s}{\tau 3}[T]\left[{ }^{3} O_{2}\right] \\
\frac{d S_{1}}{d t}=-\frac{1}{\tau 1}\left[S_{1}\right]+v \rho \sigma_{p s a}\left[S_{0}\right] \\
\frac{d[T]}{d t}=-\frac{\eta_{30}}{\tau 3}[T]-\frac{\alpha s}{\tau 3}[T]\left[{ }^{3} O_{2}\right]+\frac{\eta_{13}}{\tau 1}\left[S_{1}\right] \\
\frac{d\left[^{3} O_{2}\right]}{d t}=-\frac{\alpha s}{\tau 3}[T]\left[{ }^{3} O_{2}\right]+\frac{\eta_{0}}{\tau 0}\left[{ }^{1} O_{2}\right]+P \\
\frac{d\left[{ }^{1} O_{2}\right]}{d t}=-k p b\left[S_{0}\right]\left[{ }^{1} O_{2}\right]-k c x[R]\left[{ }^{1} O_{2}\right]-k s c[C]_{i}\left[{ }^{1} O_{2}\right]-\frac{\eta_{0}}{\tau_{0}}\left[{ }^{1} O_{2}\right]+\frac{\alpha s}{\tau 3}[T]\left[{ }^{3} O_{2}\right] \\
\frac{d[R]}{d t}=-k c x\left[{ }^{1} O_{2}\right][R]+U
\end{gathered}
$$

\section{RESULTS AND DISCUSSION}

According to the previous section, Mie's theory was employed to obtain the gold NPs optical properties at the appropriate wavelength of interest. Several types of NPs were analysed, and different concentrations were considered. Specifically the following parameters were taken, as they correspond to compatible experimental values: $40 \mathrm{~nm}$ and 80 $\mathrm{nm}$ radius, as well as NPs concentrations of $2 \cdot 10^{9}$ and $4 \cdot 10^{9} \mathrm{~cm}^{-3}$. A homogeneous distribution in a nodular basal cell carcinoma was assumed. The carcinoma optical properties were obtained at the wavelengths of interest to excite the photosensitizer molecules and trigger the photochemical reactions during PDT [14]. In this case, the interest is focused on Porphyrin-based photosensitizers, whose excitation wavelengths are in the range from 630-650 $\mathrm{nm}$. Light distribution in the tumor tissue with and without NPs embedded was obtained taking into account the previously mentioned excitation wavelengths of Porphyrin, including also some around, by means of the Monte Carlo method. A usual beam radius of $0.25 \mathrm{~cm}$ was considered, with a normalized irradiance (usual clinical value) of $200 \mathrm{~mW} / \mathrm{cm}^{2}$. Some of the 
results of optical distribution appear in Figure 1. Figure 1 represents optical absorption in the nodular basal cell carcinoma when it is treated by PDT with a Porphyrin based conventional photosensitizer. Particularly Figure 1a) represents a case in which no NPs were added. The comparison with Figure 1b), were Au NPs ( $\left.80 \mathrm{~nm}, 4 \cdot 10^{9} \mathrm{~cm}-3\right)$ were added, shows the difference in optical absorption. The addition of NPs alter the optical distribution according to the new absorption and scattering properties of the medium. Consequently, with a homogenous distribution of nanoparticles, the absorption is significantly increased in the superficial area of the tumor, revealing the increased NPs absorption. This fact prevents light penetration in the tumor, a point of extreme relevance in the application of PDT, so further attention should be paid.

a)

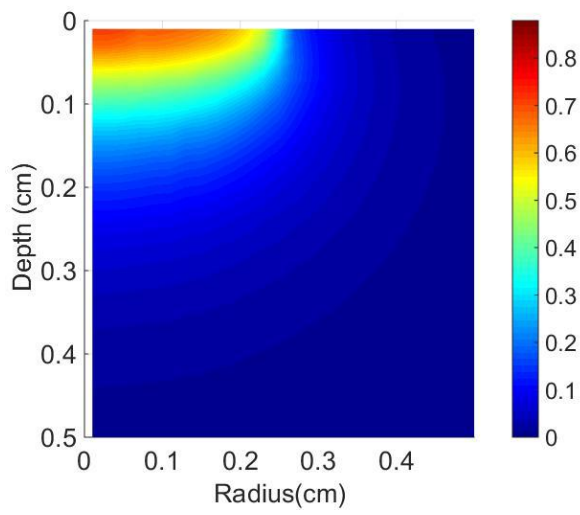

b)

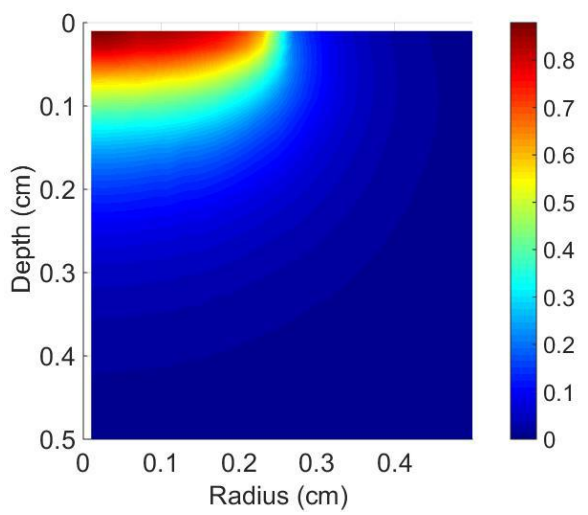

Figure 1. Optical absorption $\left(\mathrm{W} / \mathrm{cm}^{3}\right)$ in the nodular basal cell carcinoma when it is exposed to PDT with a Porphyrin based conventional photosensitizer (a) and with Au NPs $\left(80 \mathrm{~nm}, 4 \cdot 10^{9} \mathrm{~cm}^{-3}\right)$ to deliver it (b).

The influence of nanoparticle size, concentration and even light wavelength should also be considered, in order to evaluate changes in penetration depth. Penetration depth implies the possible appearance of tumor recurrence in the clinical application of PDT. One of the advantages of the use of NPs is supposed to be based on the easier spatial distribution of the photosensitizer. However, the effect on light penetration is fundamental. Evaluating light penetration numerically is not a direct question. One way to estimate it consists of employing the so-called effective penetration depth, $\delta=1 / \mu_{\text {eff }}$, where $\mu_{\text {eff }}=\sqrt{3 \mu_{a} \mu_{s}^{\prime}}$ is the effective attenuation coefficient in the diffusion limit. Table 1 shows the results of effective penetration depth with no NPs, and several types of NPs, as a function of radiation wavelength.

Table 1. Effective penetration depths for a nodular basal cell carcinoma and several NPs types.

\begin{tabular}{|c|c|c|c|}
\hline \multicolumn{4}{|c|}{ EFFECTIVE PENETRATION DEPTH } \\
\hline \multicolumn{2}{|l|}{ No NPs } & \multicolumn{2}{|c|}{$2 \cdot 10^{9} \mathrm{~cm}^{-3}$, radius $40 \mathrm{~nm}$} \\
\hline $600 \mathrm{~nm}$ & $0.0939 \mathrm{~cm}$ & $600 \mathrm{~nm}$ & $0.0933 \mathrm{~cm}$ \\
\hline $650 \mathrm{~nm}$ & $0.1290 \mathrm{~cm}$ & $650 \mathrm{~nm}$ & $0.1283 \mathrm{~cm}$ \\
\hline $700 \mathrm{~nm}$ & $0.1521 \mathrm{~cm}$ & $700 \mathrm{~nm}$ & $0.1517 \mathrm{~cm}$ \\
\hline \multicolumn{2}{|c|}{$2 \cdot 10^{9} \mathrm{~cm}^{-3}$, radius $80 \mathrm{~nm}$} & \multicolumn{2}{|c|}{$4 \cdot 10^{9} \mathrm{~cm}^{-3}$, radius $80 \mathrm{~nm}$} \\
\hline $600 \mathrm{~nm}$ & $0.0851 \mathrm{~cm}$ & $600 \mathrm{~nm}$ & $0.0779 \mathrm{~cm}$ \\
\hline $650 \mathrm{~nm}$ & $0.1169 \mathrm{~cm}$ & $650 \mathrm{~nm}$ & $0.1069 \mathrm{~cm}$ \\
\hline $700 \mathrm{~nm}$ & $0.1445 \mathrm{~cm}$ & $700 \mathrm{~nm}$ & $0.1376 \mathrm{~cm}$ \\
\hline
\end{tabular}


The dependence of the effective penetration depth with NPs size, concentration and wavelength is obvious from the previous results of Table 1. As expected, an increased concentration provokes a reduced penetration depth. Bigger NPs are also more prone to shield optical radiation traversing the tumor.

The clinical relevance of these results is complete when they are considered in relationship with the photodynamic dose [15]. This fact allows the establishment of the photodynamic treatment depth, $d_{T F D}$. Although there are several options to be considered, photodynamic depth is usually assumed to be approximately two times the effective penetration depth. The application of the previously shown photochemical model gives an explicit estimation of the photodynamic area and even volume. These results are most in agreement with the previously referred assumption.

This analysis reassures the fact that the inclusion of gold NPs as photosensitizer carriers reduces the effective light penetration depth. This is caused mainly by the increased optical absorption in the first layers of the tumor. As they could be other effects in the NPs distribution, not considered in this work, an adequate balance should be achieved in clinical praxis. The tools presented in this work may help to adequately plan PDT treatment on a particular patient and on a particular lesion with a particular geometry [16]. The potential adequacy of employing NPs could also be evaluated.

\section{CONCLUSIONS}

The use of NPs in clinical treatment and diagnostic approaches is increasing. The potentiality of PDT to selectively treat and destroy tumoral tissue has been exposed, as long as the main issue regarding its application, that is, the limited penetration depth. NPs are thought to be able to improve photosensitizer distribution, but little attention is paid to the influence in light propagation.

This work has presented a predictive model, which was used to study the effect of gold NPs on the depth of the photodynamic treatment for different wavelengths and NPs sizes and concentrations. The results show that an adequate selection of NPs size and concentration, as long as wavelength, is fundamental in order not to significantly decrease penetration depth. Is this occurs, the risk of tumor recurrence increases, and therefore the PDT treatment becomes inefficient. The tools employed, based on a complex photochemical approach, may help to estimate this fact. Consequently, they could be used in treatment planning for a particular patient, and for a particular lesion to be treated.

\section{ACKNOWLEDGEMENTS}

This work has been partially supported by the project "New active phases in transition metals and rare earth nano-oxides stabilized at high pressure" (MAT2015-69508-P) of the Spanish Ministry of Economy and Competitiveness, cofunded by FEDER funds, and by the San Cándido Foundation.

\section{REFERENCES}

[1] Vo-Dinh, T., [Biomedical Photonics Handbook], CRC Press, Boca Raton (2015).

[2] Salas-García, I., Fanjul-Vélez, F. and Arce-Diego, J. L., "Spatial photosensitizer fluorescence emission predictive analysis for photodynamic therapy monitoring applied to a skin disease," Optics Communications 285, 1581-1588 (2012).

[3] Hamblin, M. R. and Mroz, P., [Advances in Photodynamic Therapy: Basic, Translational and Clinical, Engineering in medicine \& Biology], Artech House, Norwood, USA (2008).

[4] Bechet, D., Couleaud, P., Frochot, C., Viriot, M. L., Guillemin, F. and Barberi-Heyob, M., "Nanoparticles as vehicles for delivery of photodynamic therapy agents," Trends in Biotechnology 26(11), 612-621 (2008).

[5] Feldheim, D. L., Foss, C. A., [Metal Nanoparticles Synthesis, Characterization, and Applications], Marcel Dekker, Inc., New York (2002).

[6] Salas-García, I., Fanjul-Vélez, F., Ortega-Quijano, N., Lavín-Castanedo, A., Mingo-Ortega, P., López-Escobar, M. and Arce-Diego, J. L., "Effect of gold nanoparticles in the local heating of skin tumors induced by phototherapy," Proc. SPIE 8092, 8092041-8092047 (2011). 
[7] Fanjul-Vélez, F. and Arce-Diego, J. L., "Modeling thermotherapy in vocal cords novel laser endoscopic treatment," Lasers in Medical Science 23, 169-177 (2008).

[8] Wieder, M. E., Hone, D. C., Cook, M. J., Handsley, M. M., Gavrilovic, J. and Russell, D. A., "Intracellular photodynamic therapy with photosensitizer-nanoparticle conjugates: cancer therapy using a 'Trojan horse'," Photochem. Photobiol. Sci. 5, 727-734 (2006).

[9] Oo, M. K. K., Yang, X., Du, H. and Wang, H., "5-aminolevulinic acid-conjugated gold nanoparticles for photodynamic therapy of cancer," Nanomedicine 3(6), 777-786 (2008).

[10] Bohren, C. F. and Huffman, D. R., [Absorption and Scattering of Light by Small Particles], John Wiley, New York (1983).

[11] Mätzler, C., [MATLAB Functions for Mie Scattering and Absorption], Universitas Bernensis (2002).

[12] Salas-García, I., Fanjul-Vélez, F. and Arce-Diego, J. L., "Spatial photosensitizer fluorescence emission predictive analysis for photodynamic therapy monitoring applied to a skin disease," Optics Communications 285, 1581-1588 (2012).

[13] Salas-García, I., Fanjul-Vélez, F. and Arce-Diego, J. L., "Superficial radially-resolved fluorescence and threedimensional photochemical time-dependent model for Photodynamic Therapy," Optics Letters 39, 1845-1848 (2014).

[14] Salomatina, E., Jiang, B., Novak, J. and Yaroslavsky, A. N., "Optical properties of normal and cancerous human skin in the visible and near-infrared spectral range," Journal of Biomedical Optics 11, 0640261-0640269 (2006).

[15] Salas-García, I., Fanjul-Vélez, F. and Arce-Diego, J. L., "Photosensitizer absorption coefficient modelling and necrosis prediction during photodynamic therapy," Journal of Photochemistry and Photobiology B: Biology 114, 79-86 (2012).

[16] Salas-García, I., Fanjul-Vélez, F. and Arce-Diego, J. L., "Influence of the human skin tumor type in Photodynamic Therapy analysed by a predictive model," International Journal of Photoenergy 2012, 759205 (2012). 